\title{
Positron Emission Tomography-Computed Tomography (PET-CT) in Head and Neck Pathology
}

\author{
${ }^{1}$ Luis A. Tamara, ${ }^{2}$ Ines Velez \\ ${ }^{1}$ Veterans Administration Medical Center, Houston, Texas, USA \\ ${ }^{2}$ College of Dental Medicine, Nova Southeastern University, Florida, USA \\ E-mail: luis.tamara@VAMC.gov; ivelez@nova.edu \\ Received August 20, 2011; revised September 18; accepted September 27, 2011
}

\begin{abstract}
Cancer of the head and neck is often devastating and the morbidity associated with its treatment is substantial. Positron Emission Tomography-Computed Tomography (PET-CT) combines the power of biological / molecular imaging with the anatomic detail of CT in order to provide a very sensitive and specific imaging tool for the evaluation of head and neck pathology. PET can aid the clinician in establishing diagnosis, staging, (It has been shown to be more accurate than CT), assessing, prognosis and determining response to therapy. Lymphoma, melanoma, multiple myeloma, leukemia, salivary gland tumors, odontogenic carcinomas, soft tissue sarcomas, thyroid, parathyroid, lacrimal gland and bone / cartilage tumors are some of the entities where PET-CT may be useful. (Tumors of salivary glands and of odontogenic tissue are particularly difficult to diagnose due to the relative infrequency when compared with other tumors and the extremely vast histologic variation). It is important to note that carcinoma metastasis, is the most common malignancy found within the mandibular bone. PET-CT and skeletal scintigraphy are both very sensitive and specific in these types of patients.
\end{abstract}

Keywords: PET-CT, Positron Emission Tomography, Head and Neck Cancer.

\section{Introduction}

The National Cancer Institute estimates than more than 11 million Americans with history of cancer are alive. 1.500 .000 new cancers are expected to be diagnosed each year, as well as more than 1.500 cancer deaths per day [1]. More than one person per minute dies of cancer in the US. Malignancy accounts for 1 of every 4 deaths in America. These statistics did not include basal cell and squamous cell carcinoma of the skin [1].

The complex anatomy and histology of the head and neck makes diagnosis and treatment of the area very challenging. Cancer of the head and neck is often devastating and the morbidity associated with its treatment substantial. In an effort to achieve the best possible results, specialists caring for head and neck patients should be well versed in all the available diagnostic and therapeutic modalities. Positron Emission Tomography- Computed tomography (PET-CT) is a highly sensitive and specific tool that can aid the clinician in establishing diagnosis, staging, assessing prognosis and determining response to therapy.
By far, the most common ca ncer in head and neck, excluding basal cell carcinoma, is squamous cell carcinoma (SCC). It is the $9^{\text {th }}$ most common type of cancer. Within the oral cavity, SCC has 50\% five-year death rate [1]

\section{Patho-Biology of Cancer .}

Cancer is the result of a long process that occurs from a complicated combination of genetic and non genetic factors. Therefore, carcinogenic factors are influenced by synergistic exposures and susceptibility of the host. Tumors arise from transformation of a single cell with genetic defects, into a clone. The precursor cells are the most vulnerable to carcinogenic injury to the genes, giving place to modifications of DNA, (oncogenes) which prevent the cells from normal functioning and normal reproduction mechanisms. Tumor suppressor genes (TS$\mathrm{G})$ produce proteins that are able to repair the damage of the DNA and in some cases they cause apoptosis of cells. Malignant cells lose the capability of apoptosis and, for that reason; they produce self-perpetuating molecules and exhibit mitotic activity indefinitely, resulting in the 
formation of malignant growths. The mutations accumulate, and give rise to malignant tumors which are composed of heterogeneous population of cells, with different genetic aberrations. The strongest tumoral cell is the source of a large portion of the tumor and will make it radio-resistant and / or chemo-resistant.

Malignant cells produce innumerable chemical products that, forming an abnormal molecular signaling network, are able to cause important changes in the tissue. The lethality of a tumor is related to these molecules. The chemical communication between cells is responsible for the characteristics of each type of cancer and for the complex and difficult process of metastasis. There is multilateral molecular signaling between tumoral and non-tumoral cells and the extra-cellular matrix. Among many other cancer products, there are growth factors, transcription factors, signaling molecules, neuro-endocrine substances, mitogenetic proteins, proteolytic enzymes, and angiogenetic products [2].

This ability of multi-chemical production is the hallmark of malignancy and therefore diagnosis and treatment may be based on the metabolic function of the neoplasm.

The potential to metastasize is one of the most important characteristics of malignancy. Basically, the cell involved in the metastatic process, experiences a long journey characterized by numerous obstacles. Malignant cells are separated from the primary tumor; they adhere to a blood or lymphatic vessel wall, invade through the wall between the endothelial cells and travel along the blood stream defending themselves against the immune system's components. At the end of the journey, they transport viably to a distant organ and start reproducing in the new environment and forming another tumor. Angiogenesis is induced, so there is enough nutritional supply to sustain expanding growth. The whole process requires numerous molecular cell products in order to be able to degrade physiologic barriers, stimulate replication, avoid immune surveillance, activate angiogenesis, etc.

Collections of distant manifestations that result from the substances produced by the tumor or in reaction to the tumor are called para-neoplastic syndromes. These manifestations may present in any organ or system such as endocrine, neuromuscular, musculoskeletal, cardiovascular, cutaneous, hematological, gastrointestinal, reproductive, renal and respiratory and may be the first manifestation of the tumor. 75\% of cancer patients experience a para-neoplastic disorder. (Figure 1)

Medical surveillance is of primary importance for cancer patients not only because of the metastatic potential

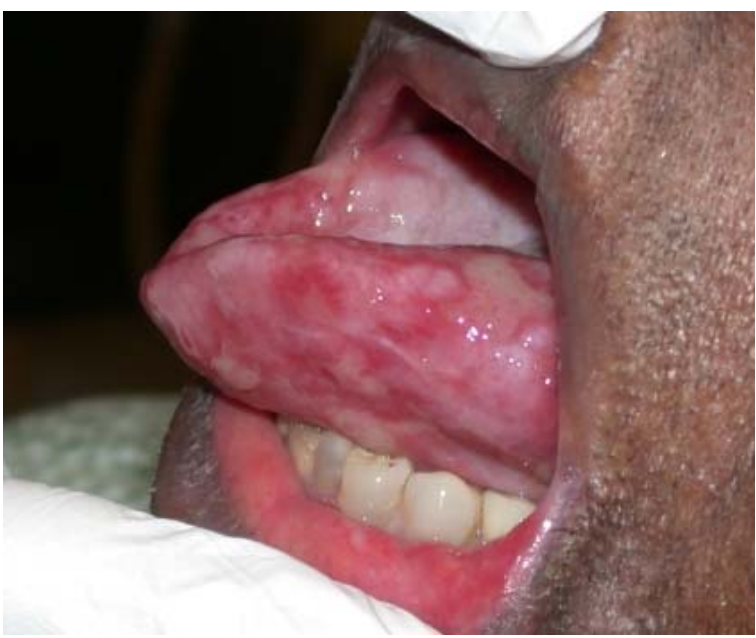

Figure 1. Para-neoplastic pemphigus in a patient with nonHodgkin lymphoma.

but also because it is known that a patient with history of cancer is at higher risk (14\%) than the general population, for developing multiple primary cancers. These are new tumors that are biologically distinct from the original primary malignancy. The risk of developing subsequent cancers depends on genetic susceptibility, immunologic condition, exposures such as chemicals and microorganisms, and carcinogenic effects of cancer treatments.

Medical surveillance is of paramount importance to cancer patients, due to the possibility of metastases and the higher risk for developing subsequent primary malignancies. PET / CT is one of the best method for surveillance as the whole body can be visualized in a single test.

The main biochemical characteristic of a malignancy is the aerobic glycolysis (the Warburg effect). Hypoxic tumor cells are highly metabolic and glucose avid, as it becomes the primary source of ATP production [3]. This is one of the basic properties of the tissue that makes PET / CT (F18-FDG) useful in cancer diagnosis and staging.

PET / CT Imaging with F-18 FDG relies on this glucose analogue's ability to be incorporated into the cell via glucose transporters and such transport is mediated by GLUT receptors. Once inside the cell F-18-FDG is phosphorylated by a system of hexokinases but undergoes no further oxidation as it fails to be recognized by the remainder of the glucose oxidation pathway. In this way it becomes "trapped" inside the cell thus allowing for imaging. Both malignant and activated inflammatory cells demonstrate upregulation of GLUT receptor and hexokinases. This fact constitutes the basis for the ability 
to differentiate between normal cells and abnormal cells such as malignant or inflammatory. (Figure 2)

\section{Squamous Cell Carcinoma.}

Squamous cell carcinoma SCC is a malignant proliferation of squamous epithelial cells. This malignancy is generated within the basal cell layers of the epithelium. Anatomically, squamous epithelium covers the external surface of the body and lines internal cavities. This tissue is also lining some of the structures that communicate internal organs with the exterior [4]. SCC comprises about $95 \%$ of oral cancers; it is the most common oro-pharyngeal / naso-pharyngeal malignancy (Figure 3), and it is the second most common cancer of the skin. (Figure 4)

Patients with tumors of paranasal sinuses usually present at advanced stage and therefore, prognosis is extremely poor. (Figure 5) PET-CT is the most sensitive way to detect these tumors and its metastases.

There are, centrally in mandible and maxilla, remnants of odontogenic epithelium called rests of Malassez. These odontogenic rests, as well as the squamous epithelium that lines numerous types of cysts, can suffer squamous metaplasia and later give origin to squamous cell carcinoma. (Figure 6)

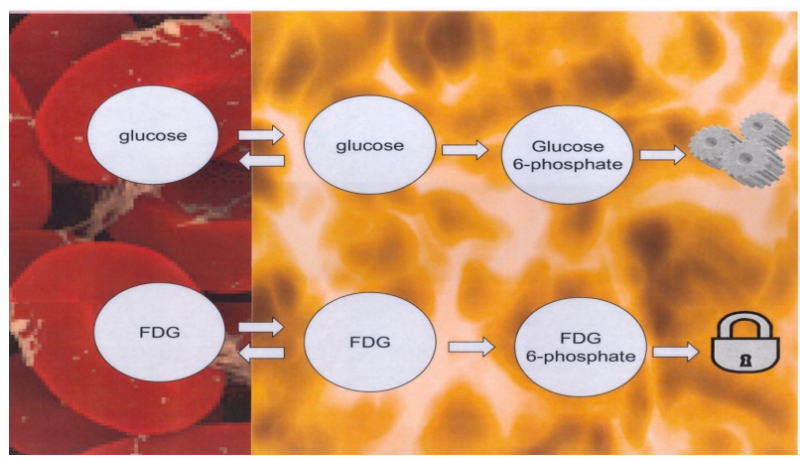

Figure 2. Glucose continues the oxidation pathway within the cells. FDG undergoes no further oxidation.

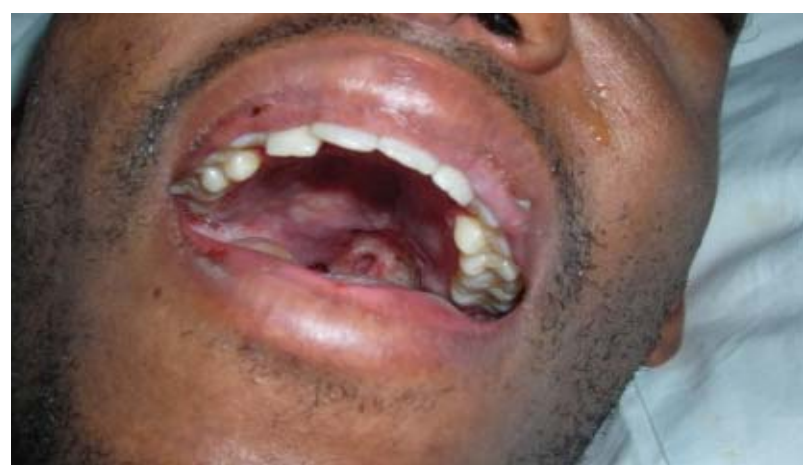

Figure 3. Nasopharyngeal carcinoma.

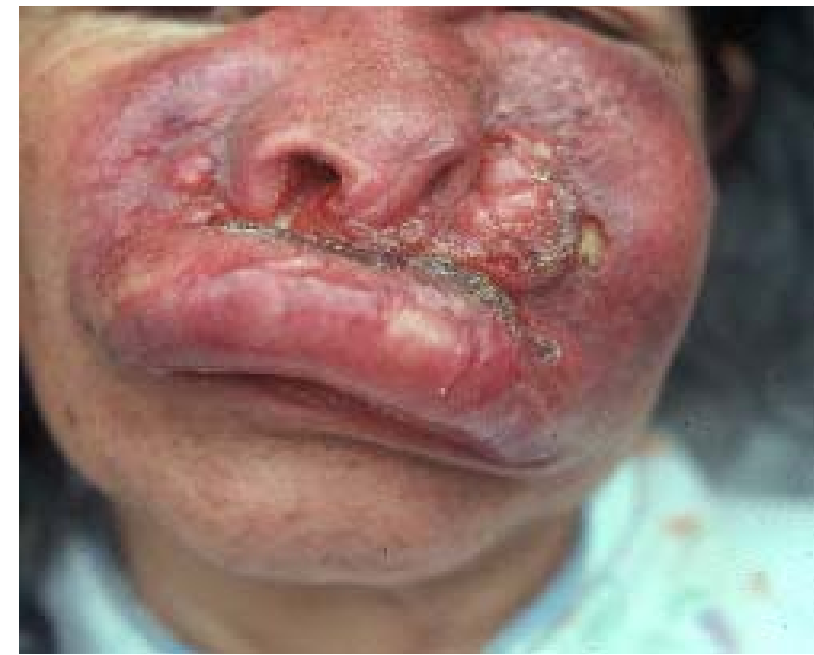

Figure 4. Squamous cell carcinoma of the skin.

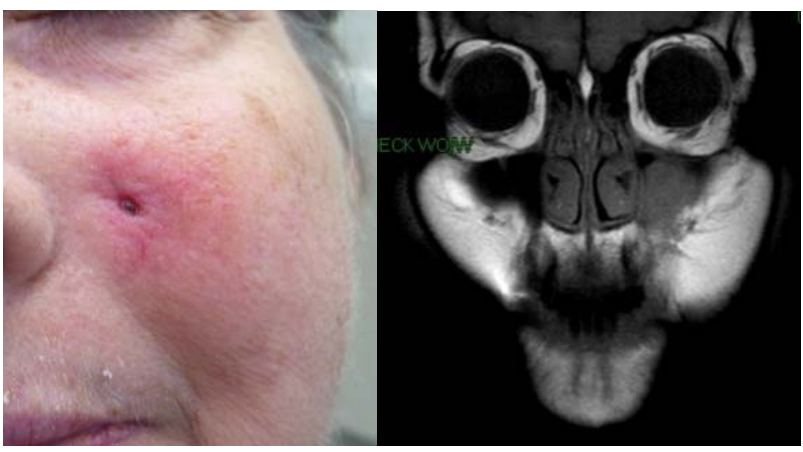

(a)

(b)

Figure 5. (a) Squamous cell carcinoma of left maxillary sinus- invading through the skin; (b) Same patient. MRI Left maxillary sinus mass.

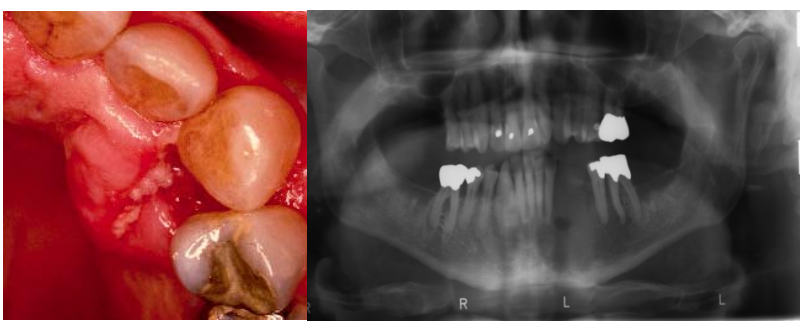

(a)

(b)

Figure 6 (a) Intra-osseous squamous cell carcinoma originated in Malazess rests; (b) Intra-osseous squamous cell carcinoma originated in an odontogenic cyst

SCC may appear in the conjunctiva and the nasolacrimal duct, where it is diagnosed early because obstruction symptoms and early metastasis to the nasal cavity.

The etiology of SCC is multi-factorial; carcinogenic factors, synergistic exposures and susceptibility of the 
host are responsible for the development of this type of tumor. There are multiple genetic defects, such as those found in syndromes, which give the host the susceptibility for malignancies. Numerous immune system conditions, from immunodeficiency to autoimmune disease, also play a role in the development of squamous cell carcinoma. Chemicals such as tobacco and alcohol, microorganisms particularly HPV 16-18-32-33-35 and radiation have been identified as being associated with oral cancer. The type of carcinogen, the frequency of exposure, the dose and the length of time it interacted with the tissue, relate to the development of this condition. This is usually a disease of the elderly [4].

Oral squamous cell carcinoma can have extreme variable clinical manifestations depending on several factors such as location, differentiation and time of evolution. The clinical appearance may vary from a flat superficial lesion (Figure 7) to a huge ulcerated destructive and invasive mass. (Figure 8) SCC may appear white or red, be flat or nodular, be crater-form or verrucous, (Figure 9a-b) exophytic or endophytic, be large or small, ulcerated or not, fast or slow-growing, be indurated or not, painful or painless. Weight loss is a common finding and according to the location, nasal obstruction, epistaxis and dysphagia are seen. Nasopharyngeal carcinoma is a type of undifferentiated squamous cell carcinoma with extremely poor prognosis. Imaging manifestations of SCC are variable, most of the time a diffuse destructive process. (Figure 10)

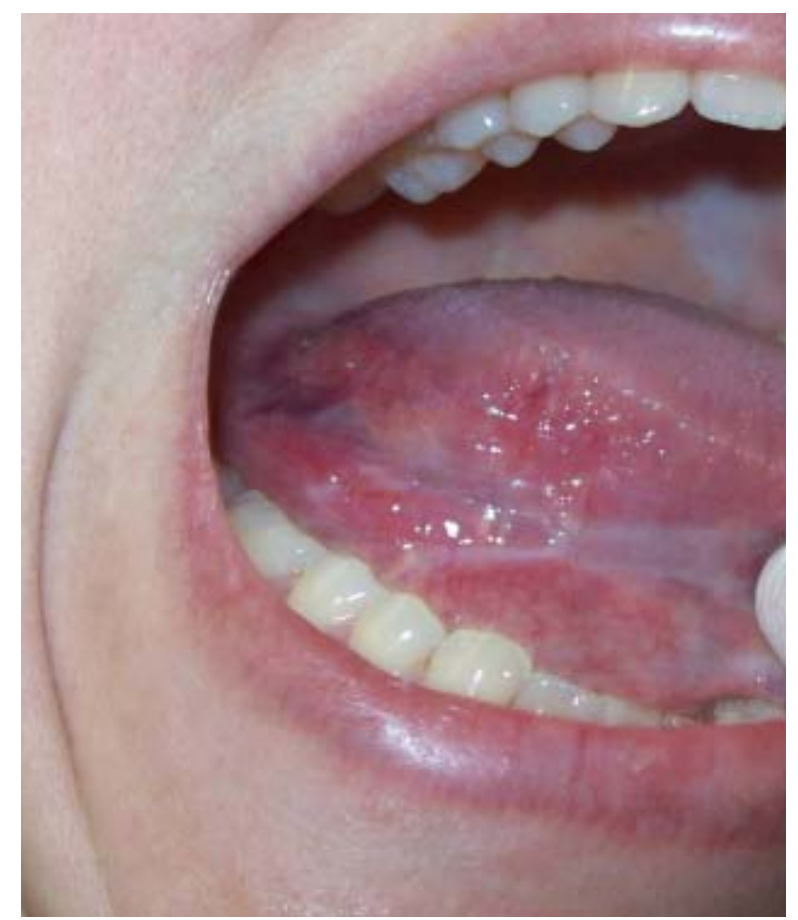

Figure 7. Early squamous cell carcinoma. Innocuous-looking lesion.

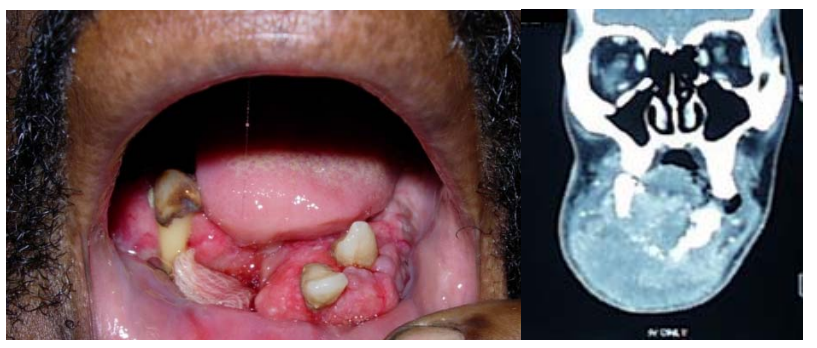

(a)

(b)

Figure 8 (a) Huge ulcerated destructive and invasive squamous cell carcinoma; (b) Same case. Destructive and invasive mass.

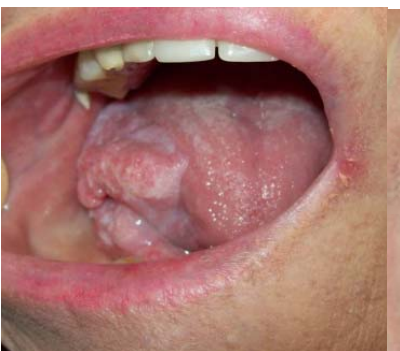

(a)

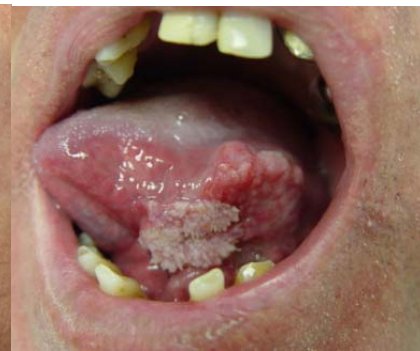

(b)
Figure 9 (a) Squamous cell carcinoma. Fast growing verrucous mass; (b) Verrucous squamous cell carcinoma associated to an invasive tumor.

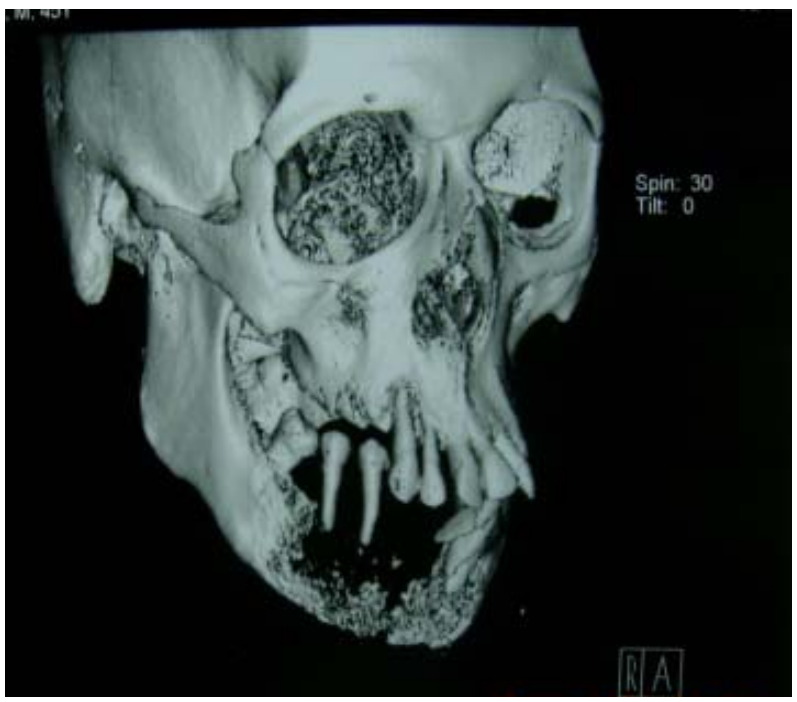

Figure 10. 3D image. Diffuse resorption of bone.

Patients with squamous cell carcinoma of the head and neck may be at higher risk of having more than one primary cancer. Field Cancerization refers to multiple areas of epithelial malignant change in the oral cavity or pharynx, (Figure 11) probably due to prolonged exposure to carcinogens such as tobacco and alcohol. Genetic susceptibility factors, immunodeficiency, immunosuppres- 
sive therapy, radiation and spread of virus, especially HPV are some of the risks factors for multiple primary squamous cell carcinomas of the head and neck [5].

Proliferative verrucous leukoplakia is a field cancerization condition, of unknown etiology, which has the unique quality of relentless transformation through different stages of hyperkeratosis, verrucous hyperplasia, verrucous carcinoma and eventually invasive carcinoma. These lesions tend to develop all over the oral mucosa. (Figure 12)

The malignant tumors' ability of multi-chemical production and signaling between the tissues is responsible for most of the cancer symptoms, such as weight loss, anemia, failure of the immune system, metastasis and death. Some patients may also die from complications of the surgery, chemo or radiotherapy. It is estimated that about one patient per hour die from oral squamous cell carcinoma in the United States.

Furthermore, every known type of cancer has the possibility of being found on head and neck [2]. Aside from squamous cell carcinoma, lymphoma, melanoma of skin and mucosa, multiple myeloma, leukemia, salivary gland tumors, odontogenic carcinomas, soft tissue sarcomas, thyroid, parathyroid, lacrimal gland and bone / cartilage tumors are some of the entities where PET-CT may be useful [7-9]. It is important to note that carcinoma metastasis, from thyroid, breast, lung, prostate, uterus or kidney, is the most

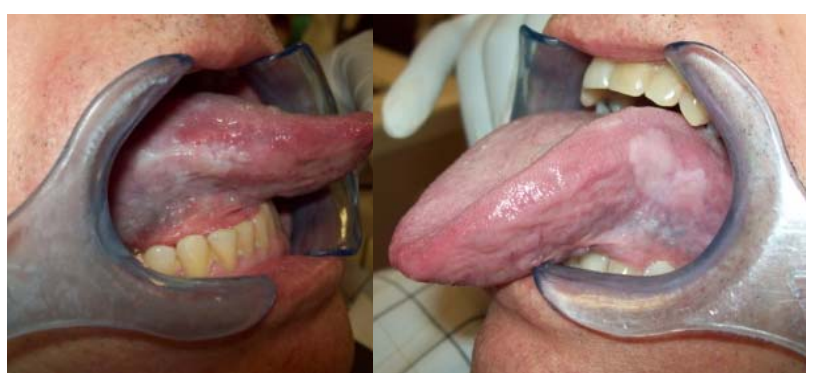

(a)

(b)

Figure 11. (a) Squamous cell carcinoma of the right la- teral border of the tongue; (b) Left lateral tongue. This lesion appeared simultaneously with the lesion on the right side.

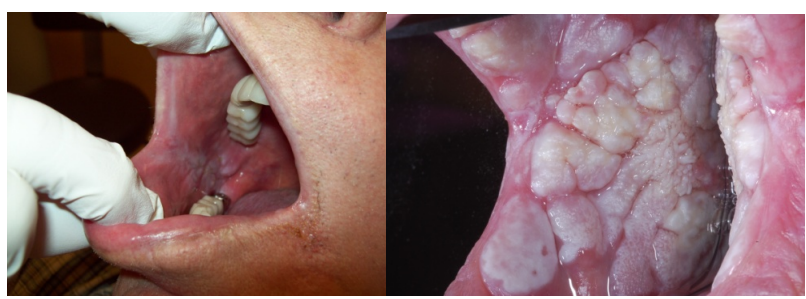

(a)

(b)

Figure 12. (a) White slightly elevated lesion. Histologic diagnosis: hyperkeratosis; (b) Same patient. Two years later. Histologic diagnosis: verrucous carcinoma. common malignancy found within the mandibular bone. PET-CT is also very sensitive and specific in these types of patients. (Figure 13 and Figure 14) .

It has been found that 75.000 newly diagnosed cases and 21,000 deaths from lymphoma occur every year. Extra -nodal Non Hodgkin lymphomas are present in head and neck more frequently than in any other site. The mucosa, at the junction of the hard and soft palate, is the most common place, followed by the Waldeyer's ring. These lymphomas are almost always B-cell lymphomas and are part of the so called MALTOMAS (mucosal associated lymphoid tissue tumors). MALTOMAS may also arise, among other areas, in stomach, lung, parotid and lacrimal glands.

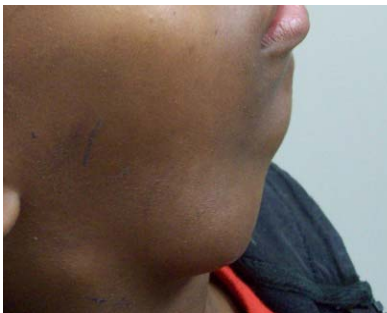

(a)

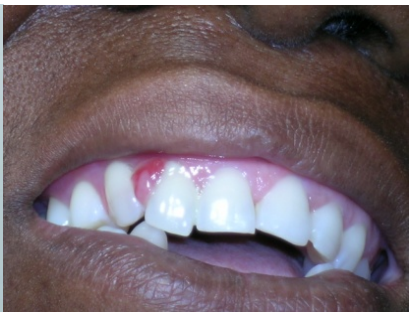

(b)
Figure 13. (a) Hodgkin lymphoma. Several enlarged lymph nodes cervical chain; (b) MALT lymphoma extension from palate.

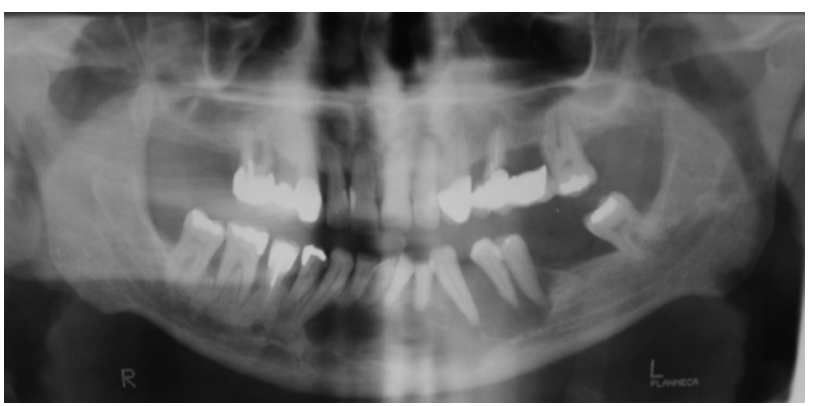

Figure 14. Panoramic radiography showing bone destruction by lymphoma.

Lymphoma, melanoma of skin and mucosa, multiple myeloma, salivary gland tumors, odontogenic carcinomas, soft tissue sarcomas, thyroid, parathyroid, lacrimal gland and bone / cartilage tumors are some of the entities where PET-CT may be useful. It is important to note that carcinoma metastasis, is the most common malignancy found within the mandibular bone. PET-CT is both very sensitive and specific in these types of patients.

MALTOMAS are also a special consideration for the head and neck specialist due to the association with pre- 
existent immune diseases, with head and neck manifestations, such as Sjogren syndrome and Hashimoto thyroiditis. On the other hand, Hodgkin lymphoma which is initially a nodal disease, frequently exhibits the first manifestations in cervical lymphatic chains.

Sarcoma is a general term applied to malignancies arising from mesenchymal tissue. Due to the increasing understanding of human cells and tissue, some tumors believed to be "sarcomas" are not originated in mesenchymal tissue. Fibrous and adipose tissue, skeletal muscle, smooth muscle, nerve fibers, bone and cartilage, vessels, blood, hemopoietic and lymphatic tissues, are present in the head and neck and may give origin to sarcomas.

Melanoma, one of the most aggressive cancers, has the ability to metastasize into any organ. Near 70.000 cutane ous melanoma cases are diagnosed per year. Mucosal melanomas metastasize more frequently and faster than skin melanomas and have an extremely poor prognosis. They are not rare in the upper oral mucosa and in the eye.

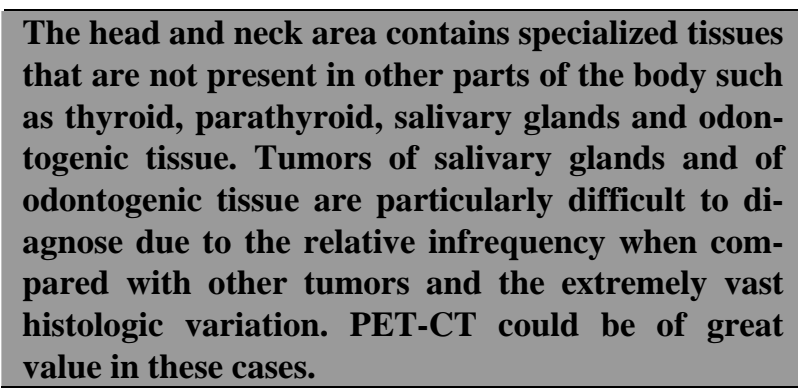

The progress in diagnosis and early staging of cancer, as well as the improvement on treatment, reflects an increased survival rate. As the survivor population increases due to the evolving nature of the scientific field, medical surveillance is of paramount importance and PET-CT is one of the best tools for this purpose.

Among the most common primary sites of patients with multiple cancers are head and neck areas, especially oro-pharynx with squamous cell carcinoma, and NonHo- dgkin lymphoma. Oro-pharyngeal cancer exhibits the largest Estimated Absolute Risk (EAR) for subsequent cancers in male and female patients ${ }^{1}$. Skin and mucosal melanoma and leukemia are primary malignant entities associated with moderate risk of multiple cancers. Secondary / multiple malignancies are effectively found with PET-CT SCAN.

PET-CT is of paramount importance in clinical staging, and has been shown to be more accurate than CT. It makes a significant difference identifying malignant normal size nodes.
Initial staging is indispensable for the choice of therapy and the assessment of the prognosis, and it is based on the size of the primary tumor and the metastasis. There are several staging systems. The most known the TNM system which evaluates T: the size of the primary tumor, $\mathrm{N}$ : absence or presence of regional lymph node metastasis and M: distant metastasis. PET CT is of paramount importance in clinical staging, and has been shown to be more accurate than CT. It makes a significant difference identifying malignant normal size nodes.

Accurate staging is indispensable to define the type of surgery and the area of the radiotherapy field. Schwartz et al. reported sensitivity of $96 \%$ and specificity of 98.5\% for detection of nodal metastasis during the initial staging of SCC with FDG-PET / CT.

Cancer of unknown primary constitutes one of the most difficult cases for the clinician. PET / CT should be performed as the initial test in cases of unknown primary and the biopsies should be directed according to $\mathrm{PET} / \mathrm{CT}$ results.

One of the main advantages of FDG-PET / CT during the initial staging of head and neck cancer is its capacity to identify distant disease, metastatic or synchronous, in any part of the body, all in the same test [10]. FDG-PET / CT is also of paramount importance for treatment planning. FDG uptake is a prognostic indicator. Tumors with high FDG uptake have poorer prognosis and higher recurrence rate as compared with tumors with low FDG uptake. One advantage is that PET-CT data can be imported into the radiation treatment planning computer for precise radiation dose and volume.

Surgery remains the best therapy for squamous cell carcinoma. Sometimes combination with radiotherapy and chemotherapy is indicated. Removal of the entire lesion with its draining lymphatics and lymph node chains, including the tissues contained in the same area of the neck, (neck dissections) are needed in many cases of head and neck carcinoma. Sometimes the dissection includes the tail of the parotid, the sternocleidomastoid muscle, the spinal accessory nerve and the mandibular branch of the facial nerve. These surgeries leave variable, often severe, levels of functional compromise and deformities for life. It has been suggested by Kovacs et al [10] that patients with positive lymph nodes in FDG-PET / CT, should undergo neck dissection and the sentinel lymph node biopsy technique should be performed in patients with negative FDG-PET / CT.

It is also important to have in mind, that cases of carcinoma of unknown primary are not rare in the head and neck (2\% of head and neck cancer diagnosis). [11] The 
most common sites for the primary neoplasm are the tonsillar area and the base of tongue. The treatment, which causes significant morbidity, consists of widefield radiation that covers pharynx, larynx and bilateral neck. If the primary is localized, risks of complications decrease considerably. FDG-PET / CT is useful in identification of the primary site. Wong et al published that in $27 \%$ of cases of unknown primary carcinoma, after negative CT, MRI and endoscopy, the primary tumor was localized with FDG-PET / CT. This fact has a significant impact regarding the morbidity and mortality. Menda and Graham [12] (2005) stated that PET / CT should be performed as the initial test in cases of unknown primary and the biopsies should be directed according to PET / CT results. Additional metastases are also discovered by PET-CT which may change the treatment and / or the radiation field [13]. On the other hand, the most common intraosseous malignancy in the mandibular bones is metastasis of carcinoma specially from lung, uterus, kidney, thyroid and prostate. These malignancies may be identified by PET-CT.

Patients with unresectable cancers are initially treated with chemo-radiation. Cumulative data show that PET / CT has resulted in improved evaluation of response to therapy both in the curative and neoadjuvant settings with relatively high sensitivity, specificity, positive and negative predictive values. Interpretative pitfalls can be found in the presence of inflammatory response, tumor stunning and adjunct therapies. When considering a change in management strategies, histologic confirmation of PET / CT findings may be appropriate in selected cases [13].

\section{In summary, why PET / CT in the evalua- tion of head and neck cancer?}

Within the last decade, PET-CT has exploded into the c linical arena as a powerful imaging tool for the evaluation of many malignancies. Head and neck malignancies are no exception and PET-CT consistently performs better than the standard anatomic imaging techniques CT and MRI.

The reasons for this superior performance are multiple and can be outlined as follows:

1) PET-CT can localize primary head and neck malignancies that have not been identified by clinical / endoscopic exam or other imaging techniques. (Figure 15)

2) PET-CT can assess response to therapy more accurately than CT or MRI. It is not as affected by anatomical distortion from prior surgery or radiation therapy. It relies on biological tumoral activity rather than size changes. (Figure 16)

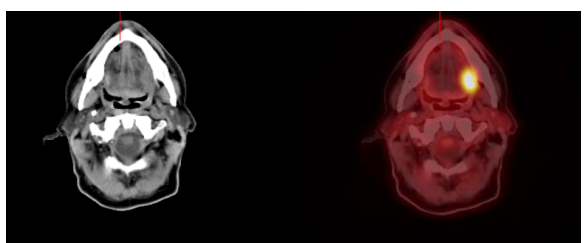

Figure 15. SCCA UNC Chapel Hill Department of nuclear medicine. SCCA of tongue. PET-CT can identify metastatic lymph node involvement in normal size nodes.

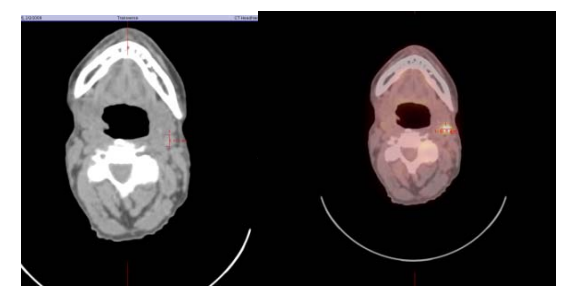

Figure 16. SCCA UNC Chapel Hill Department of nuclear medicine. PET allows more accurate staging. If this patient had been staged with CT alone, he would have been under staging.

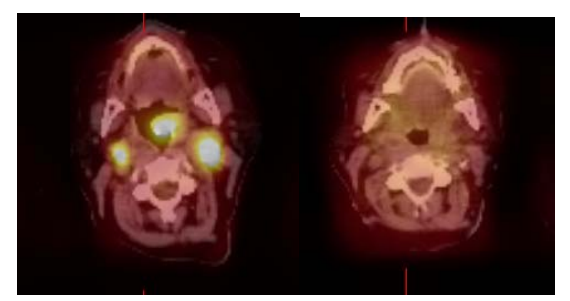

Figure 17. SCCA UNC Chapel Hill Department of nuclear medicine.

3) PET-CT can be used in radiation therapy planning for precise target delineation of the biological tumor volume. In this regard PET-CT consistently outperforms dedicated CT imaging. (Figure 17)

4) PET-CT combines the power of biological / molecular imaging with the anatomic detail of CT in order to provide a very sensitive and specific imaging tool for the evaluation of head and neck malignancies.

PET-CT combines the power of biological / molecular imaging with the anatomic detail of CT in order to provide a very sensitive and specific imaging tool for the evaluation of head and neck malignancies. The use of PET-CT in head and neck cancer may make a significant difference in the treatment and prognosis of many patients.

\section{References}

[1] American Cancer Society. Cancer facts \& figures 2009. 
Atlanta: American Cancer Society, 2009.

[2] D.E. James, R. Brown, J. Navarro, P.R. Pilch. "Insulin regulatable tissues express a unique insulin-sensitive glucose transport protein”. Nature 333: 183-185, 1988. doi:10.1038/333183a0

[3] D.E. James, M. Strube , M. Mueckler. "Molecular cloning and characterization of an insulin regulatable glucose transporter”. Nature 338: 83-87, 1989. doi:10.1038/338083a0

[4] M.H. Ross, E.J. Reith. "Epithelium” in: Histology. New York, Ed Harper \& Row: 1985

[5] C.B. Begg. "Methodological and Statistical Considerations in the Study of Multiple Primary Cancers". In: Neugut AI, Meadows AT, Robimson E, Multiple Primary cancers. Philadelphia, PA: Lippincott Williams and Wilkins; 1999:13-26

[6] R. Marx, D. Stern. "Premalignant and malignant epithelial tumors of mucosa and skin”. In: Oral and Maxillofacial Pathology. Quintaesscence, First edition, 2003; 283 -373.

[7] F. R. Miller, D. Hussey, M. Beeram, T. Eng, H.S. McGuff, A.O.Randall. "Positron Emission tomography in the management of unknown primary head and neck carcinoma”. Archives of Otolaryngology Head and neck Surgery.131:626-629. 2005. doi:10.1001/archotol.131.7.626

[8] H. Schoder, H.W.D. Yeung, M. Gonen, D. Krauss, S.M. Larson: "Positron Emission imaging of head and neck cancer, including thyroid carcinoma." Seminars in $\mathrm{Nu}$ clear Medicine. 34:180-197. 2004. doi:10.1053/j.semnuclmed.2004.03.004
[9] A.F. Kovacs, N. Dobert, J.Gaa.: "Positron emission tomography in combination with sentinel lymph node biopsy reduces the rate of elective neck dissections in the treatment of oral and oropharyngeal cancer". Journal of Clinical Oncology 22:3973-3980, 2004. doi:10.1200/JCO.2004.01.124

[10] D.L. Schwartz, E. Ford, J. Rajendran, B. Yueh, M. D. Coltrera, F. Virgin, et al "FDG-PET / CT imaging for preradiotherapy staging of head and neck squamous cell carcinoma”. International Journal of Radiation Oncology Biology and Physics 61:129-136, 2005. doi:10.1016/j.ijrobp.2004.03.040

[11] W.J. Wong, M. Saunders: "The impact of FDG PET on the management of occult primary head and neck tumors”. Clinical Oncology 15:461-466. 2003. doi:10.1016/j.clon.2003.07.006

[12] Y. Menda, M. Graham. "Update on FDG / PET and Positron emission tomography / computed tomography imaging of squamous head and neck cancers”. Seminars in Nuclear Medicine 35:214-219. 2005. doi:10.1053/j.semnuclmed.2005.05.001

[13] L. Kostakoglu. "FDG-PET Evaluation of response to treatment”. PET Clinics. 3: 37-75. 2008. doi:10.1016/j.cpet.2008.09.001

[14] G.W. Goerres, D.T. Schimdt, F. Bandhauer. "Positron emission tomography in the early follow up of advanced head and neck cancer". Archives of Otolaryngology Head and Neck Surgery 130: 105-109. 2004. doi:10.1001/archotol.130.1.105 